\title{
A Longitudinal Pilot Study to Evaluate Non-visual Icons in a Mobile Exertion Application
}

\author{
Huimin Qian, Ravi Kuber, and Andrew Sears \\ UMBC, 1000 Hilltop Circle, Baltimore, MD 21250, USA \\ \{huimin1, rkuber, asears\} @umbc . edu
}

\begin{abstract}
This paper describes an evaluation of a mobile exertion application, developed to promote physical activity among adult users. To extend previous work, we aimed to identify changes in walking behavior over time, and to determine the efficacy of non-visual cues presented via the phone. Data was gathered using three methods: automated logging, participant-maintained diaries and interviews. Findings revealed that participants were able to respond most effectively to multimodal icons to modify their pace over a two week period. However, their preferences were noted to change depending on the situational context. The study has also highlighted the complexities associated with data collection techniques for mobile evaluations.
\end{abstract}

\section{Introduction}

Mobile persuasive applications, such as MPTrain [4] and StepMan [1] have been developed to address the challenges faced remaining motivated to keep fit. However, these are often evaluated at one fixed point in time, under controlled conditions (e.g. lab-based). In contrast, longitudinal studies can provide a more comprehensive overview of usage and an insight into behavioral patterns. Examples include the threeweek evaluation of Ubifit Garden [3], where the researchers were able to monitor the type and duration of activities conducted, and how these varied over time. Reis et al. [6] were able to identify the contexts in which modalities were selected for use when using mobile technologies. User preference was found to change depending on the environment, and other factors such as lightning, movement, and hand usage.

In this paper, we briefly describe the development of a mobile exertion application designed to aid users to maintain and modify levels of pace, through the presentation of non-visual icons. To extend previous work [5], and as a first step towards understanding user behavior, a pilot study was conducted where participants used the application over a two week period in locations of their choice. The aim was to identify the following:

- Which types of feedback are most commonly used to maintain levels of physical activity when walking in uncontrolled environments?

- Does choice of feedback vary depending on environment and/or context?

- How do participants behave in response to signals rendered via different modalities? 


\section{Design of Mobile Application and Pilot Study}

A pedometer-style application has been developed for the Nokia N95 mobile telephone. The device is strapped to the waist using a belt. As the user walks around his/her environment, the number of steps are calculated using data gathered from the built-in accelerometer. An optimal level of pace is determined, based on the user's step rate. Auditory, tactile or multimodal (composed of both auditory and tactile effects) prompts (all played at $158 \mathrm{~Hz}$ ) are presented via the phone, to assist the user in maintaining his/her desired rate of pace by encouraging him/her to modify pace as appropriate. To move faster, the user is presented with icons lasting for $200 \mathrm{~ms}$, while to move slower, $800 \mathrm{~ms}$ icons are played. The non-visual cues are presented every six seconds until the user reaches their target rate of pace. All auditory information was presented at $65 \mathrm{~dB}$. To extend our previous work, a study was performed to determine the efficacy of non-visual cues over a two week period while traversing a variety of terrains, rather than being constrained to a controlled environment, such as in [5]. We also aimed to identify whether over time, users would react faster to modify their level of pace.

\section{Methodology}

Three adults ( 2 male, 1 female), aged between 50 and 60, were selected from a local community center. No difficulties were reported with levels of hearing or mobility. All participants classified themselves as moderately physically active. However, the time spent weekly on walking would depend on work schedules and other commitments. Participants were each provided with thirty minutes of training with the application, during which time their pace was recorded when walking at a comfortable speed $(\mathrm{N})$. This enabled us to determine a desired level of pace $(\mathrm{N} *$ $120 \%$ ). Non-visual icons would be presented by the application, if participants were unable to maintain their individual target paces. Participants were then asked to use the mobile application at any point over the two week period, and record their walking goals and experience of using the application in an online diary. The distance and time taken for each journey, rate of pace, and how effectively users were able to maintain this pace was automatically logged by our software. We envisaged that recording data in a diary would aid us in gaining a deeper understanding of their walking behavior, by providing an insight into key events within the journey and reasons behind the selection of feedback. It would also reduce the risk of problems associated with recalling past events, as these would be noted immediately after each trial. Online diaries could also be monitored by the evaluator without disturbing the users.

At the end of the first and second week of use, the participants were interviewed to follow-up on events logged within the diaries and to obtain step rate data stored on each mobile handset. The interviews focused on which types of feedback had been used, key events that disrupted any walk, frequency of use, levels of workload experienced, and if participants had faced any difficulties with the application. 


\section{Results and Discussion}

Ten trials were logged by the three participants, lasting between seven to thirty-five minutes in duration (Table 1). All trials were conducted in outdoor environments, where visual distractions were present (e.g. traffic) on a range of terrains including both flat and uneven ground (e.g. grass, inclines etc).

Table 1. Results from Study

\begin{tabular}{|c|c|c|c|}
\hline Trial & Duration (Mins) & Cue & Terrain \\
\hline 1 & 30 & Multimodal & Forest trail \\
\hline 2 & 35 & Tactile & Uneven terrain \\
\hline 3 & 10 & Multimodal & Near home environment \\
\hline 4 & 10 & Multimodal & Uneven terrain \\
\hline 5 & 7 & Multimodal & Uneven terrain \\
\hline 6 & 9 & Multimodal & Uneven terrain \\
\hline 7 & 15 & Tactile & Uneven terrain \\
\hline 8 & 20 & Audio & Near home environment \\
\hline 9 & 20 & Multimodal & Near home environment \\
\hline 10 & 20 & Multimodal & Near home environment \\
\hline
\end{tabular}

Multimodal icons were self-selected for presentation in seven out of the ten trials. When asked to discuss reasons behind the selection of these cues, participants suggested that combining auditory and tactile feedback together was found to offer a more "noticeable" alert in inclement environments. Participant \#1 suggested as the sound of traffic became louder, the audio was more difficult to process, but he was still able to perceive the tactile cues presented while keeping his eyes free to monitor the presence of cars in close proximity to his route. When in quiet environments (e.g. back yard), Participant \#3 suggested that she was able to use auditory feedback. However, she suggested in unfamiliar surroundings more feedback would be needed, as more concentration would be spent navigating the paths. It appeared that context and awareness of the environment played a role in selection.

In order to identify how effectively participants were able to react to the non-visual feedback presented, the number of instances when icons were played were calculated for each trial. A total of 120 instances of feedback were presented (11 audio icons, 31 tactile icons and 78 multimodal icons). The majority of feedback was presented towards the beginning of each trial $(>85 \%)$, mostly aiming to prompt participants to walk faster. This was expected, as participants noted that they would try to walk faster than their comfortable pace, but it was difficult to gauge from memory, what this rate would be. To determine the accuracy of responses to feedback presented, the number of correct responses to the cues, was divided by the total number of six second intervals within each journey. For all walking trials, response accuracy rates were higher than $97 \%$, demonstrating that participants were able to react to all forms of feedback effectively. Due to the small sample of participants, it is difficult to draw conclusions as to whether perception improved during the time period. In all ten trials, the participants walked faster than their desired target pace for certain periods of time. When asked for the reasons behind walking faster than necessary, 
participants suggested that in order to divide attention between the non-visual cues and the surrounding environment, it was thought to be less cognitively intensive to walk faster. Participant \#1 mentioned that he tended to walk faster deliberately to avoid the delivery of signals. However, no participants reported experiencing levels of overload from presentation of the icons.

While participants did not make as much use of the application as initially envisaged, results provided us with awareness of the contexts and environments in which different forms of feedback proved to be useful. In a future study, we aim to enhance the application, to modify the type of feedback presented during each trial, to provide more flexibility when environments change (e.g. when moving from quiet to noisy surroundings). We will then use the longitudinal study to evaluate how users respond to the feedback presented over time.

\section{Practicalities of Conducting the Pilot Study}

While laboratory-based studies offer an effective way to gather data, conclusions derived from studies may only pertain to performing tasks under constrained conditions. Diary studies were selected as one method to gather data when performing mobile tasks, as it was thought that participants could undertake activities in the field, and would be able to describe their goals and actions with minimal intervention from the evaluators. By being able to view online diaries, it was noticeable that few entries were made. It also required effort to understand the meaning associated with the comments made. As a result, the evaluators needed to contact the participants to follow-up on certain points. It also proved challenging to cross-reference entries from the diary with the steps taken, as comments were not as extensive as anticipated. Certain events were omitted as they were thought to be trivial by participants, also mentioned by [2]. Findings from our study suggested that follow-up interviews provided a richer representation of events, than relying on the diary study alone.

\section{References}

1. Bieber, G., Diener, H.: StepMan - A New Kind of Music Interaction. In: Human Computer Interaction International (2005)

2. Brandt, J., Weiss, N., Klemmer, S.R.: txt418r: Lowering the Burden for Diary Studies under Mobile Conditions. In: CHI EA 2007, pp. 2303-2308. ACM Press, New York (2008)

3. Consolvo, S., McDonald, D.W., Toscos, T., et al.: Activity Sensing in the Wild: A Field Trial of Ubifit Garden. In: CHI 2008, pp. 1797-1806. ACM Press, New York (2008)

4. Oliver, N., Flores-Mangas, F.: MPTrain: A Mobile Music and Physiology Based Personal Trainer. In: MobileHCI 2006. ACM Press, New York (2006)

5. Qian, H., Kuber, R., Sears, A.: Maintaining levels of activity using a haptic personal training application. In: CHI EA 2010, pp. 3217-3222. ACM Press, New York (2010)

6. Reis, T., Sá, M.D., Carriço, L.: Multimodal Interaction: Real Context Studies on Mobile Digital Artefacts. In: Pirhonen, A., Brewster, S. (eds.) HAID 2008. LNCS, vol. 5270, pp. 60-69. Springer, Heidelberg (2008) 